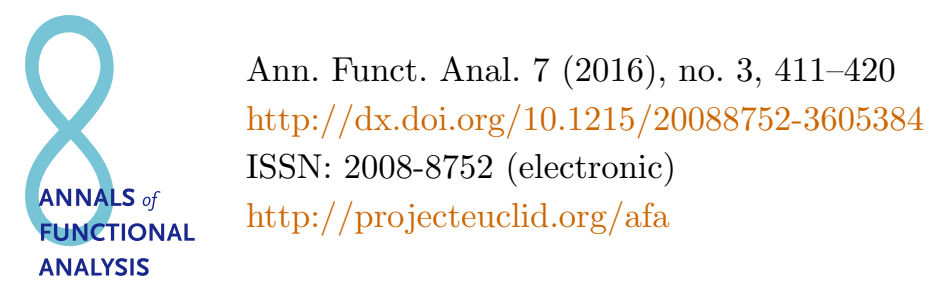

\title{
A BOUNDED TRANSFORM APPROACH TO SELF-ADJOINT OPERATORS: FUNCTIONAL CALCULUS AND AFFILIATED VON NEUMANN ALGEBRAS
}

\author{
CHRISTIAN BUDDE and KLAAS LANDSMAN \\ Communicated by Q.-W. Wang
}

\begin{abstract}
Spectral theory and functional calculus for unbounded self-adjoint operators on a Hilbert space are usually treated through von Neumann's Cayley transform. Using ideas of Woronowicz, we redevelop this theory from the point of view of multiplier algebras and the so-called bounded transform (which establishes a bijective correspondence between closed operators and pure contractions). This also leads to a simple account of the affiliation relation between von Neumann algebras and self-adjoint operators.
\end{abstract}

\section{INTRODUCTORY OVERVIEW}

The theory of unbounded self-adjoint operators on a Hilbert space was initiated by von Neumann [7], partly motivated by mathematical problems of quantum mechanics. The monograph by Schmüdgen [10] presents an excellent survey of the present state of the art.

Von Neumann's approach was based on the Cayley transform, and in its subsequent development the notion of a spectral measure played an important role, especially in defining a functional calculus. We consider this route a bit indirect and will avoid both by first invoking the bounded transform instead of the Cayley transform; that is, the formal expressions

$$
\begin{aligned}
& S=T{\sqrt{I+T^{2}}}^{-1}, \\
& T=S{\sqrt{I-S^{2}}}^{-1},
\end{aligned}
$$

Copyright 2016 by the Tusi Mathematical Research Group.

Received Jul. 20, 2015; Accepted Dec. 10, 2015.

2010 Mathematics Subject Classification. Primary 47B25; Secondary 46L10.

Keywords. bounded transform, self-adjoint operators, von Neumann algebras. 


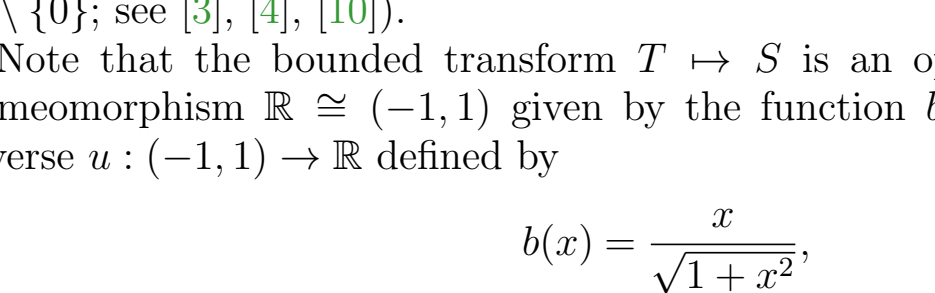

\title{
A BOUNDED TRANSFORM APPROACH TO SELF-ADJOINT OPERATORS: FUNCTIONAL CALCULUS AND AFFILIATED VON NEUMANN ALGEBRAS
}

\author{
CHRISTIAN BUDDE and KLAAS LANDSMAN \\ Communicated by Q.-W. Wang
}

\begin{abstract}
Spectral theory and functional calculus for unbounded self-adjoint operators on a Hilbert space are usually treated through von Neumann's Cayley transform. Using ideas of Woronowicz, we redevelop this theory from the point of view of multiplier algebras and the so-called bounded transform (which establishes a bijective correspondence between closed operators and pure contractions). This also leads to a simple account of the affiliation relation between von Neumann algebras and self-adjoint operators.
\end{abstract}

\section{INTRODUCTORY OVERVIEW}

The theory of unbounded self-adjoint operators on a Hilbert space was initiated by von Neumann [7], partly motivated by mathematical problems of quantum mechanics. The monograph by Schmüdgen [10] presents an excellent survey of the present state of the art.

Von Neumann's approach was based on the Cayley transform, and in its subsequent development the notion of a spectral measure played an important role, especially in defining a functional calculus. We consider this route a bit indirect and will avoid both by first invoking the bounded transform instead of the Cayley transform; that is, the formal expressions

$$
\begin{aligned}
& S=T{\sqrt{I+T^{2}}}^{-1}, \\
& T=S{\sqrt{I-S^{2}}}^{-1},
\end{aligned}
$$

Copyright 2016 by the Tusi Mathematical Research Group.

Received Jul. 20, 2015; Accepted Dec. 10, 2015.

2010 Mathematics Subject Classification. Primary 47B25; Secondary 46L10.

Keywords. bounded transform, self-adjoint operators, von Neumann algebras. 


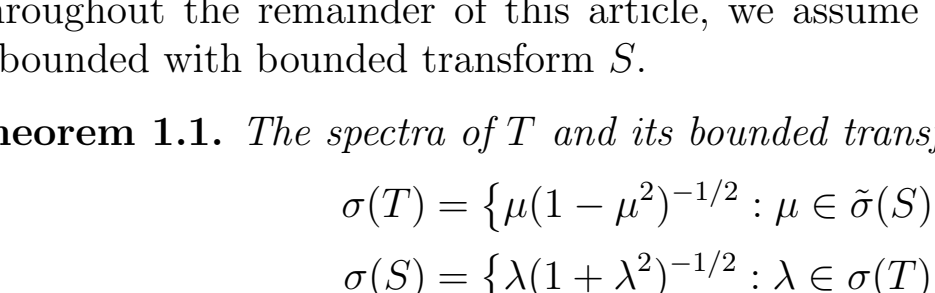

\title{
A BOUNDED TRANSFORM APPROACH TO SELF-ADJOINT OPERATORS: FUNCTIONAL CALCULUS AND AFFILIATED VON NEUMANN ALGEBRAS
}

\author{
CHRISTIAN BUDDE and KLAAS LANDSMAN \\ Communicated by Q.-W. Wang
}

\begin{abstract}
Spectral theory and functional calculus for unbounded self-adjoint operators on a Hilbert space are usually treated through von Neumann's Cayley transform. Using ideas of Woronowicz, we redevelop this theory from the point of view of multiplier algebras and the so-called bounded transform (which establishes a bijective correspondence between closed operators and pure contractions). This also leads to a simple account of the affiliation relation between von Neumann algebras and self-adjoint operators.
\end{abstract}

\section{INTRODUCTORY OVERVIEW}

The theory of unbounded self-adjoint operators on a Hilbert space was initiated by von Neumann [7], partly motivated by mathematical problems of quantum mechanics. The monograph by Schmüdgen [10] presents an excellent survey of the present state of the art.

Von Neumann's approach was based on the Cayley transform, and in its subsequent development the notion of a spectral measure played an important role, especially in defining a functional calculus. We consider this route a bit indirect and will avoid both by first invoking the bounded transform instead of the Cayley transform; that is, the formal expressions

$$
\begin{aligned}
& S=T{\sqrt{I+T^{2}}}^{-1}, \\
& T=S{\sqrt{I-S^{2}}}^{-1},
\end{aligned}
$$

Copyright 2016 by the Tusi Mathematical Research Group.

Received Jul. 20, 2015; Accepted Dec. 10, 2015.

2010 Mathematics Subject Classification. Primary 47B25; Secondary 46L10.

Keywords. bounded transform, self-adjoint operators, von Neumann algebras. 


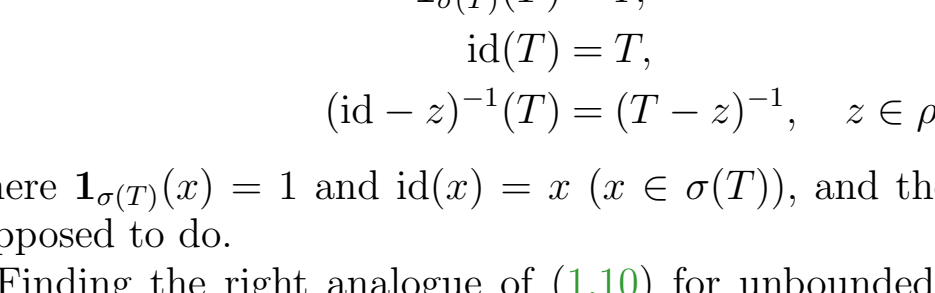

\title{
A BOUNDED TRANSFORM APPROACH TO SELF-ADJOINT OPERATORS: FUNCTIONAL CALCULUS AND AFFILIATED VON NEUMANN ALGEBRAS
}

\author{
CHRISTIAN BUDDE and KLAAS LANDSMAN \\ Communicated by Q.-W. Wang
}

\begin{abstract}
Spectral theory and functional calculus for unbounded self-adjoint operators on a Hilbert space are usually treated through von Neumann's Cayley transform. Using ideas of Woronowicz, we redevelop this theory from the point of view of multiplier algebras and the so-called bounded transform (which establishes a bijective correspondence between closed operators and pure contractions). This also leads to a simple account of the affiliation relation between von Neumann algebras and self-adjoint operators.
\end{abstract}

\section{INTRODUCTORY OVERVIEW}

The theory of unbounded self-adjoint operators on a Hilbert space was initiated by von Neumann [7], partly motivated by mathematical problems of quantum mechanics. The monograph by Schmüdgen [10] presents an excellent survey of the present state of the art.

Von Neumann's approach was based on the Cayley transform, and in its subsequent development the notion of a spectral measure played an important role, especially in defining a functional calculus. We consider this route a bit indirect and will avoid both by first invoking the bounded transform instead of the Cayley transform; that is, the formal expressions

$$
\begin{aligned}
& S=T{\sqrt{I+T^{2}}}^{-1}, \\
& T=S{\sqrt{I-S^{2}}}^{-1},
\end{aligned}
$$

Copyright 2016 by the Tusi Mathematical Research Group.

Received Jul. 20, 2015; Accepted Dec. 10, 2015.

2010 Mathematics Subject Classification. Primary 47B25; Secondary 46L10.

Keywords. bounded transform, self-adjoint operators, von Neumann algebras. 


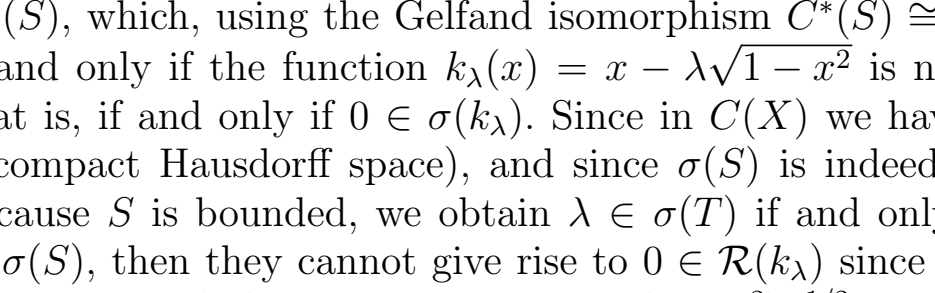

\title{
A BOUNDED TRANSFORM APPROACH TO SELF-ADJOINT OPERATORS: FUNCTIONAL CALCULUS AND AFFILIATED VON NEUMANN ALGEBRAS
}

\author{
CHRISTIAN BUDDE and KLAAS LANDSMAN \\ Communicated by Q.-W. Wang
}

\begin{abstract}
Spectral theory and functional calculus for unbounded self-adjoint operators on a Hilbert space are usually treated through von Neumann's Cayley transform. Using ideas of Woronowicz, we redevelop this theory from the point of view of multiplier algebras and the so-called bounded transform (which establishes a bijective correspondence between closed operators and pure contractions). This also leads to a simple account of the affiliation relation between von Neumann algebras and self-adjoint operators.
\end{abstract}

\section{INTRODUCTORY OVERVIEW}

The theory of unbounded self-adjoint operators on a Hilbert space was initiated by von Neumann [7], partly motivated by mathematical problems of quantum mechanics. The monograph by Schmüdgen [10] presents an excellent survey of the present state of the art.

Von Neumann's approach was based on the Cayley transform, and in its subsequent development the notion of a spectral measure played an important role, especially in defining a functional calculus. We consider this route a bit indirect and will avoid both by first invoking the bounded transform instead of the Cayley transform; that is, the formal expressions

$$
\begin{aligned}
& S=T{\sqrt{I+T^{2}}}^{-1}, \\
& T=S{\sqrt{I-S^{2}}}^{-1},
\end{aligned}
$$

Copyright 2016 by the Tusi Mathematical Research Group.

Received Jul. 20, 2015; Accepted Dec. 10, 2015.

2010 Mathematics Subject Classification. Primary 47B25; Secondary 46L10.

Keywords. bounded transform, self-adjoint operators, von Neumann algebras. 


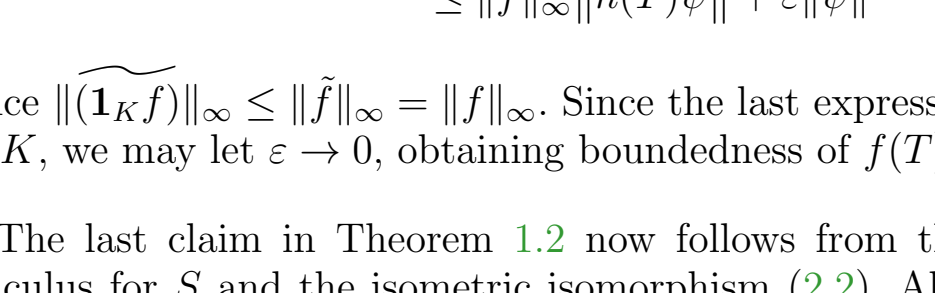

\title{
A BOUNDED TRANSFORM APPROACH TO SELF-ADJOINT OPERATORS: FUNCTIONAL CALCULUS AND AFFILIATED VON NEUMANN ALGEBRAS
}

\author{
CHRISTIAN BUDDE and KLAAS LANDSMAN \\ Communicated by Q.-W. Wang
}

\begin{abstract}
Spectral theory and functional calculus for unbounded self-adjoint operators on a Hilbert space are usually treated through von Neumann's Cayley transform. Using ideas of Woronowicz, we redevelop this theory from the point of view of multiplier algebras and the so-called bounded transform (which establishes a bijective correspondence between closed operators and pure contractions). This also leads to a simple account of the affiliation relation between von Neumann algebras and self-adjoint operators.
\end{abstract}

\section{INTRODUCTORY OVERVIEW}

The theory of unbounded self-adjoint operators on a Hilbert space was initiated by von Neumann [7], partly motivated by mathematical problems of quantum mechanics. The monograph by Schmüdgen [10] presents an excellent survey of the present state of the art.

Von Neumann's approach was based on the Cayley transform, and in its subsequent development the notion of a spectral measure played an important role, especially in defining a functional calculus. We consider this route a bit indirect and will avoid both by first invoking the bounded transform instead of the Cayley transform; that is, the formal expressions

$$
\begin{aligned}
& S=T{\sqrt{I+T^{2}}}^{-1}, \\
& T=S{\sqrt{I-S^{2}}}^{-1},
\end{aligned}
$$

Copyright 2016 by the Tusi Mathematical Research Group.

Received Jul. 20, 2015; Accepted Dec. 10, 2015.

2010 Mathematics Subject Classification. Primary 47B25; Secondary 46L10.

Keywords. bounded transform, self-adjoint operators, von Neumann algebras. 


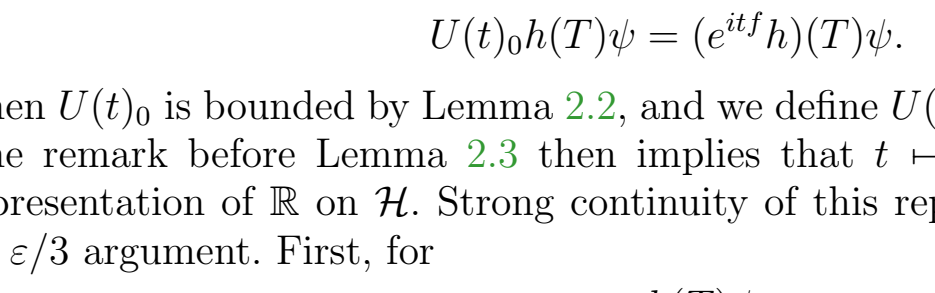

\title{
A BOUNDED TRANSFORM APPROACH TO SELF-ADJOINT OPERATORS: FUNCTIONAL CALCULUS AND AFFILIATED VON NEUMANN ALGEBRAS
}

\author{
CHRISTIAN BUDDE and KLAAS LANDSMAN \\ Communicated by Q.-W. Wang
}

\begin{abstract}
Spectral theory and functional calculus for unbounded self-adjoint operators on a Hilbert space are usually treated through von Neumann's Cayley transform. Using ideas of Woronowicz, we redevelop this theory from the point of view of multiplier algebras and the so-called bounded transform (which establishes a bijective correspondence between closed operators and pure contractions). This also leads to a simple account of the affiliation relation between von Neumann algebras and self-adjoint operators.
\end{abstract}

\section{INTRODUCTORY OVERVIEW}

The theory of unbounded self-adjoint operators on a Hilbert space was initiated by von Neumann [7], partly motivated by mathematical problems of quantum mechanics. The monograph by Schmüdgen [10] presents an excellent survey of the present state of the art.

Von Neumann's approach was based on the Cayley transform, and in its subsequent development the notion of a spectral measure played an important role, especially in defining a functional calculus. We consider this route a bit indirect and will avoid both by first invoking the bounded transform instead of the Cayley transform; that is, the formal expressions

$$
\begin{aligned}
& S=T{\sqrt{I+T^{2}}}^{-1}, \\
& T=S{\sqrt{I-S^{2}}}^{-1},
\end{aligned}
$$

Copyright 2016 by the Tusi Mathematical Research Group.

Received Jul. 20, 2015; Accepted Dec. 10, 2015.

2010 Mathematics Subject Classification. Primary 47B25; Secondary 46L10.

Keywords. bounded transform, self-adjoint operators, von Neumann algebras. 


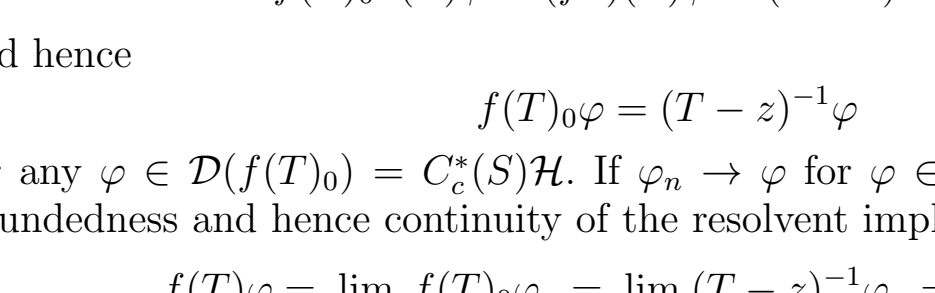

\title{
A BOUNDED TRANSFORM APPROACH TO SELF-ADJOINT OPERATORS: FUNCTIONAL CALCULUS AND AFFILIATED VON NEUMANN ALGEBRAS
}

\author{
CHRISTIAN BUDDE and KLAAS LANDSMAN \\ Communicated by Q.-W. Wang
}

\begin{abstract}
Spectral theory and functional calculus for unbounded self-adjoint operators on a Hilbert space are usually treated through von Neumann's Cayley transform. Using ideas of Woronowicz, we redevelop this theory from the point of view of multiplier algebras and the so-called bounded transform (which establishes a bijective correspondence between closed operators and pure contractions). This also leads to a simple account of the affiliation relation between von Neumann algebras and self-adjoint operators.
\end{abstract}

\section{INTRODUCTORY OVERVIEW}

The theory of unbounded self-adjoint operators on a Hilbert space was initiated by von Neumann [7], partly motivated by mathematical problems of quantum mechanics. The monograph by Schmüdgen [10] presents an excellent survey of the present state of the art.

Von Neumann's approach was based on the Cayley transform, and in its subsequent development the notion of a spectral measure played an important role, especially in defining a functional calculus. We consider this route a bit indirect and will avoid both by first invoking the bounded transform instead of the Cayley transform; that is, the formal expressions

$$
\begin{aligned}
& S=T{\sqrt{I+T^{2}}}^{-1}, \\
& T=S{\sqrt{I-S^{2}}}^{-1},
\end{aligned}
$$

Copyright 2016 by the Tusi Mathematical Research Group.

Received Jul. 20, 2015; Accepted Dec. 10, 2015.

2010 Mathematics Subject Classification. Primary 47B25; Secondary 46L10.

Keywords. bounded transform, self-adjoint operators, von Neumann algebras. 


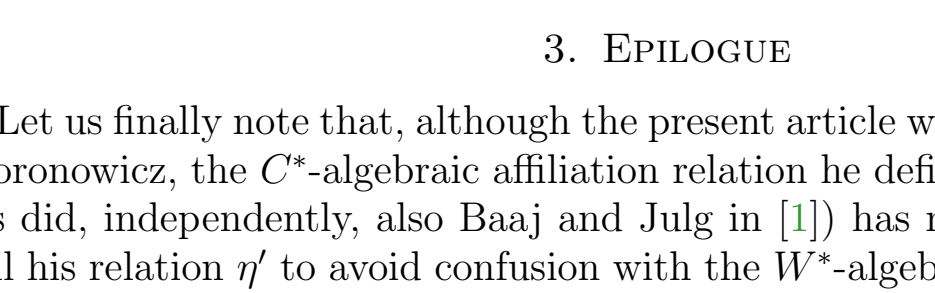

\title{
A BOUNDED TRANSFORM APPROACH TO SELF-ADJOINT OPERATORS: FUNCTIONAL CALCULUS AND AFFILIATED VON NEUMANN ALGEBRAS
}

\author{
CHRISTIAN BUDDE and KLAAS LANDSMAN \\ Communicated by Q.-W. Wang
}

\begin{abstract}
Spectral theory and functional calculus for unbounded self-adjoint operators on a Hilbert space are usually treated through von Neumann's Cayley transform. Using ideas of Woronowicz, we redevelop this theory from the point of view of multiplier algebras and the so-called bounded transform (which establishes a bijective correspondence between closed operators and pure contractions). This also leads to a simple account of the affiliation relation between von Neumann algebras and self-adjoint operators.
\end{abstract}

\section{INTRODUCTORY OVERVIEW}

The theory of unbounded self-adjoint operators on a Hilbert space was initiated by von Neumann [7], partly motivated by mathematical problems of quantum mechanics. The monograph by Schmüdgen [10] presents an excellent survey of the present state of the art.

Von Neumann's approach was based on the Cayley transform, and in its subsequent development the notion of a spectral measure played an important role, especially in defining a functional calculus. We consider this route a bit indirect and will avoid both by first invoking the bounded transform instead of the Cayley transform; that is, the formal expressions

$$
\begin{aligned}
& S=T{\sqrt{I+T^{2}}}^{-1}, \\
& T=S{\sqrt{I-S^{2}}}^{-1},
\end{aligned}
$$

Copyright 2016 by the Tusi Mathematical Research Group.

Received Jul. 20, 2015; Accepted Dec. 10, 2015.

2010 Mathematics Subject Classification. Primary 47B25; Secondary 46L10.

Keywords. bounded transform, self-adjoint operators, von Neumann algebras. 


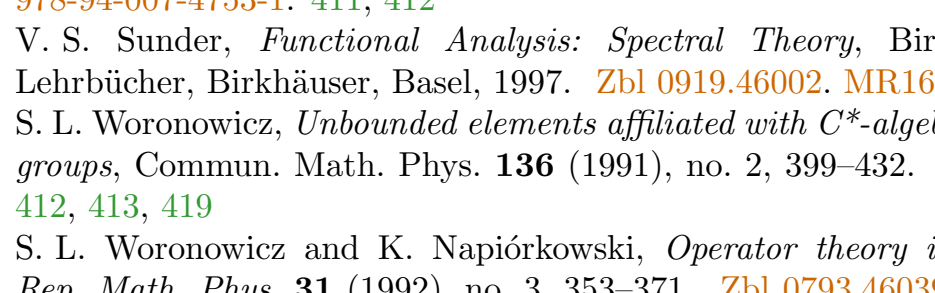

\title{
A BOUNDED TRANSFORM APPROACH TO SELF-ADJOINT OPERATORS: FUNCTIONAL CALCULUS AND AFFILIATED VON NEUMANN ALGEBRAS
}

\author{
CHRISTIAN BUDDE and KLAAS LANDSMAN \\ Communicated by Q.-W. Wang
}

\begin{abstract}
Spectral theory and functional calculus for unbounded self-adjoint operators on a Hilbert space are usually treated through von Neumann's Cayley transform. Using ideas of Woronowicz, we redevelop this theory from the point of view of multiplier algebras and the so-called bounded transform (which establishes a bijective correspondence between closed operators and pure contractions). This also leads to a simple account of the affiliation relation between von Neumann algebras and self-adjoint operators.
\end{abstract}

\section{INTRODUCTORY OVERVIEW}

The theory of unbounded self-adjoint operators on a Hilbert space was initiated by von Neumann [7], partly motivated by mathematical problems of quantum mechanics. The monograph by Schmüdgen [10] presents an excellent survey of the present state of the art.

Von Neumann's approach was based on the Cayley transform, and in its subsequent development the notion of a spectral measure played an important role, especially in defining a functional calculus. We consider this route a bit indirect and will avoid both by first invoking the bounded transform instead of the Cayley transform; that is, the formal expressions

$$
\begin{aligned}
& S=T{\sqrt{I+T^{2}}}^{-1}, \\
& T=S{\sqrt{I-S^{2}}}^{-1},
\end{aligned}
$$

Copyright 2016 by the Tusi Mathematical Research Group.

Received Jul. 20, 2015; Accepted Dec. 10, 2015.

2010 Mathematics Subject Classification. Primary 47B25; Secondary 46L10.

Keywords. bounded transform, self-adjoint operators, von Neumann algebras. 